Karma Dajani · Martijn de Vries

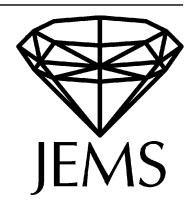

\title{
Measures of maximal entropy for random $\beta$-expansions
}

Received September 11, 2003 and in revised form January 26, 2004

Abstract. Let $\beta>1$ be a non-integer. We consider $\beta$-expansions of the form $\sum_{i=1}^{\infty} d_{i} / \beta^{i}$, where the digits $\left(d_{i}\right)_{i>1}$ are generated by means of a Borel map $K_{\beta}$ defined on $\{0,1\}^{\mathbb{N}} \times[0,\lfloor\beta\rfloor /(\beta-1)]$. We show that $K_{\beta}$ has a unique mixing measure $v_{\beta}$ of maximal entropy with marginal measure an infinite convolution of Bernoulli measures. Furthermore, under the measure $v_{\beta}$ the digits $\left(d_{i}\right)_{i \geq 1}$ form a uniform Bernoulli process. In case 1 has a finite greedy expansion with positive coefficients, the measure of maximal entropy is Markov. We also discuss the uniqueness of $\beta$-expansions.

Keywords. Greedy expansions, lazy expansions, Markov chains, measures of maximal entropy

\section{Introduction}

Let $\beta>1$ be a non-integer. There are two well-known expansions of numbers $x$ in $[0,\lfloor\beta\rfloor /(\beta-1)]$ of the form

$$
x=\sum_{i=1}^{\infty} \frac{a_{i}}{\beta^{i}}
$$

with $a_{i} \in\{0,1, \ldots,\lfloor\beta\rfloor\}$. The largest in lexicographical order is the greedy expansion ([I] $[\mathrm{R} 1],[\mathrm{R} 2])$, and the smallest is the lazy expansion ([JS], [EJK], [DK1]). The greedy expansion is obtained by iterating the greedy transformation $T_{\beta}:[0,\lfloor\beta\rfloor /(\beta-1)] \rightarrow$ $[0,\lfloor\beta\rfloor /(\beta-1)]$, defined by

$$
T_{\beta}(x)= \begin{cases}\beta x(\bmod 1), & 0 \leq x<1, \\ \beta x-\lfloor\beta\rfloor, & 1 \leq x \leq\lfloor\beta\rfloor /(\beta-1) .\end{cases}
$$

The lazy expansion is obtained by iterating the lazy transformation $S_{\beta}:[0,\lfloor\beta\rfloor /(\beta-1)]$ $\rightarrow[0,\lfloor\beta\rfloor /(\beta-1)]$, defined by

$$
S_{\beta}(x)=\beta x-d \quad \text { for } x \in \Delta(d),
$$

K. Dajani: Universiteit Utrecht, Fac. Wiskunde en Informatica and MRI, Budapestlaan 6, P.O. Box 80.000, 3508 TA Utrecht, the Netherlands; e-mail: dajani@math.uu.nl

M. de Vries: Vrije Universiteit Amsterdam, Faculty of Exact Sciences, de Boelelaan 1081, 1081 HV Amsterdam, the Netherlands; e-mail: mdvries@cs.vu.nl

Mathematics Subject Classification (2000): 28D05 
where

$$
\Delta(0)=\left[0, \frac{\lfloor\beta\rfloor}{\beta(\beta-1)}\right],
$$

and

$$
\begin{aligned}
\Delta(d) & =\left(\frac{\lfloor\beta\rfloor}{\beta-1}-\frac{\lfloor\beta\rfloor-d+1}{\beta}, \frac{\lfloor\beta\rfloor}{\beta-1}-\frac{\lfloor\beta\rfloor-d}{\beta}\right] \\
& =\left(\frac{\lfloor\beta\rfloor}{\beta(\beta-1)}+\frac{d-1}{\beta}, \frac{\lfloor\beta\rfloor}{\beta(\beta-1)}+\frac{d}{\beta}\right], \quad d \in\{1, \ldots,\lfloor\beta\rfloor\} .
\end{aligned}
$$
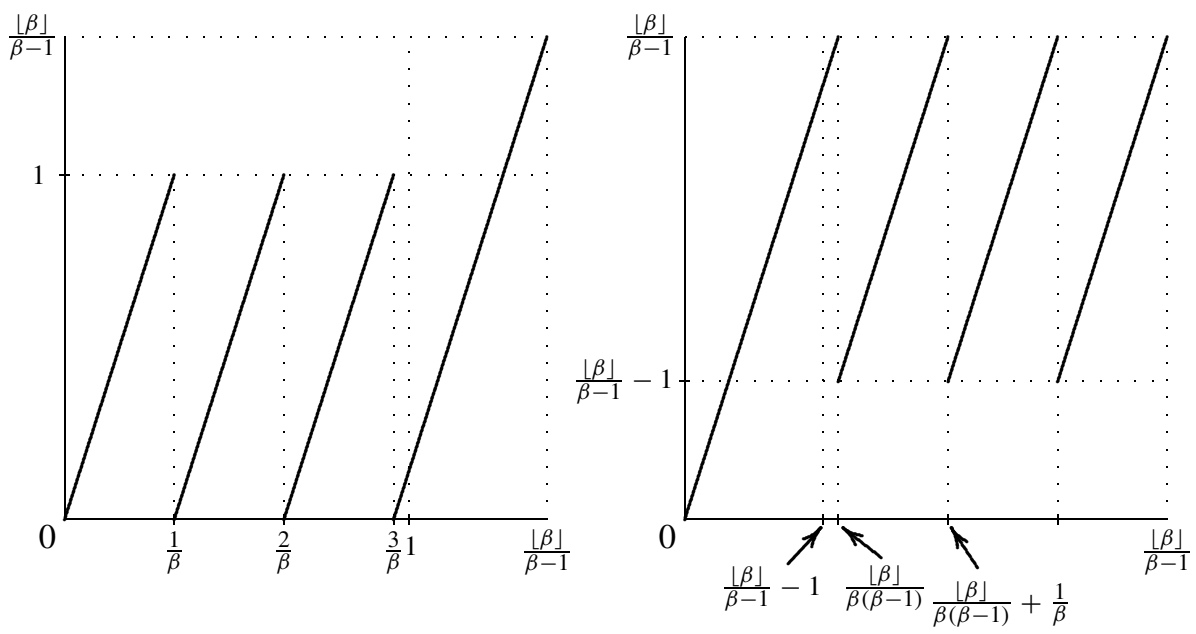

Fig. 1. The greedy map $T_{\beta}$ (left), and the lazy map $S_{\beta}$ (right). Here $\beta=\pi$.

We denote by $\mu_{\beta}$ the extended Parry measure (see $[\mathrm{P}],[\mathrm{G}]$ ) on $[0,\lfloor\beta\rfloor /(\beta-1)]$ which is absolutely continuous with respect to Lebesgue measure, and with density

$$
h_{\beta}(x)= \begin{cases}\frac{1}{F(\beta)} \sum_{n=0}^{\infty} \frac{1}{\beta^{n}} 1_{\left[0, T_{\beta}^{n}(1)\right)}(x), & 0 \leq x<1, \\ 0, & 1 \leq x \leq\lfloor\beta\rfloor /(\beta-1),\end{cases}
$$

where $F(\beta)=\int_{0}^{1}\left(\sum_{x<T_{\beta}^{n}(1)} 1 / \beta^{n}\right) d x$ is a normalizing constant.

Define $\ell:[0,\lfloor\beta\rfloor /(\beta-1)] \rightarrow[0,\lfloor\beta\rfloor /(\beta-1)]$ by

$$
\ell(x)=\frac{\lfloor\beta\rfloor}{\beta-1}-x,
$$

and consider the lazy measure $\rho_{\beta}$ defined on $[0,\lfloor\beta\rfloor /(\beta-1)]$ by $\rho_{\beta}(A)=\mu_{\beta}(\ell(A))$ for every measurable set $A$. It is easy to see $([\mathrm{DK} 1])$ that $\ell$ is a measurable isomorphism between $\left([0,\lfloor\beta\rfloor /(\beta-1)], \mu_{\beta}, T_{\beta}\right)$ and $\left([0,\lfloor\beta\rfloor /(\beta-1)], \rho_{\beta}, S_{\beta}\right)$. 
In order to produce other expansions in a dynamical way, a new $\beta$-transformation $K_{\beta}$ was introduced in [DK2]. The expansions generated by iterating this map are random mixtures of greedy and lazy expansions. This is done as follows. Superimpose the greedy map and the corresponding lazy map on $[0,\lfloor\beta\rfloor /(\beta-1)]$, one gets $\lfloor\beta\rfloor$ overlapping regions of the form

$$
S_{k}=\left[\frac{k}{\beta}, \frac{\lfloor\beta\rfloor}{\beta(\beta-1)}+\frac{k-1}{\beta}\right], \quad k=1, \ldots,\lfloor\beta\rfloor,
$$

which one refers to as switch regions. On $S_{k}$, the greedy map assigns the digit $k$, while the lazy map assigns the digit $k-1$. Outside these switch regions both maps are identical, and hence they assign the same digits. Now, define a new random expansion in base $\beta$ by randomizing the choice of the map used in the switch regions. So, whenever $x$ belongs to a switch region flip a coin to decide which map will be applied to $x$, and hence which digit will be assigned. To be more precise, partition the interval $[0,\lfloor\beta\rfloor /(\beta-1)]$ into switch regions $S_{k}$ and equality regions $E_{k}$, where

$$
\begin{aligned}
& E_{k}=\left(\frac{\lfloor\beta\rfloor}{\beta(\beta-1)}+\frac{k-1}{\beta}, \frac{k+1}{\beta}\right), \quad k=1, \ldots,\lfloor\beta\rfloor-1, \\
& E_{0}=\left[0, \frac{1}{\beta}\right), \quad E_{\lfloor\beta\rfloor}=\left(\frac{\lfloor\beta\rfloor}{\beta(\beta-1)}+\frac{\lfloor\beta\rfloor-1}{\beta}, \frac{\lfloor\beta\rfloor}{\beta-1}\right] .
\end{aligned}
$$

Let

$$
S=\bigcup_{k=1}^{\lfloor\beta\rfloor} S_{k}, \quad E=\bigcup_{k=0}^{\lfloor\beta\rfloor} E_{k},
$$

and consider $\Omega=\{0,1\}^{\mathbb{N}}$ with the product $\sigma$-algebra $\mathcal{A}$. Let $\sigma: \Omega \rightarrow \Omega$ be the left shift, and define $K_{\beta}: \Omega \times[0,\lfloor\beta\rfloor /(\beta-1)] \rightarrow \Omega \times[0,\lfloor\beta\rfloor /(\beta-1)]$ by

$$
K_{\beta}(\omega, x)= \begin{cases}(\omega, \beta x-k), & x \in E_{k}, k=0,1, \ldots,\lfloor\beta\rfloor, \\ (\sigma(\omega), \beta x-k), & x \in S_{k} \text { and } \omega_{1}=1, k=1, \ldots,\lfloor\beta\rfloor, \\ (\sigma(\omega), \beta x-k+1), & x \in S_{k} \text { and } \omega_{1}=0, k=1, \ldots,\lfloor\beta\rfloor .\end{cases}
$$

The elements of $\Omega$ represent the coin tosses ("heads" $=1$ and "tails" $=0$ ) used every time the orbit hits a switch region. Let

$$
d_{1}=d_{1}(\omega, x)= \begin{cases}k & \text { if } x \in E_{k}, k=0,1, \ldots,\lfloor\beta\rfloor, \\ & \text { or }(\omega, x) \in\left\{\omega_{1}=1\right\} \times S_{k}, k=1, \ldots,\lfloor\beta\rfloor, \\ k-1 & \text { if }(\omega, x) \in\left\{\omega_{1}=0\right\} \times S_{k}, k=1, \ldots,\lfloor\beta\rfloor .\end{cases}
$$

Then

$$
K_{\beta}(\omega, x)= \begin{cases}\left(\omega, \beta x-d_{1}\right) & \text { if } x \in E \\ \left(\sigma(\omega), \beta x-d_{1}\right) & \text { if } x \in S\end{cases}
$$


Set $d_{n}=d_{n}(\omega, x)=d_{1}\left(K_{\beta}^{n-1}(\omega, x)\right)$, and let $\pi_{2}: \Omega \times[0,\lfloor\beta\rfloor /(\beta-1)] \rightarrow$ $[0,\lfloor\beta\rfloor /(\beta-1)]$ be the canonical projection onto the second coordinate. Then

$$
\pi_{2}\left(K_{\beta}^{n}(\omega, x)\right)=\beta^{n} x-\beta^{n-1} d_{1}-\cdots-\beta d_{n-1}-d_{n},
$$

and rewriting yields

$$
x=\frac{d_{1}}{\beta}+\frac{d_{2}}{\beta^{2}}+\cdots+\frac{d_{n}}{\beta^{n}}+\frac{\pi_{2}\left(K_{\beta}^{n}(\omega, x)\right)}{\beta^{n}} .
$$

Since $\pi_{2}\left(K_{\beta}^{n}(\omega, x)\right) \in[0,\lfloor\beta\rfloor /(\beta-1)]$, it follows that

$$
x-\sum_{i=1}^{n} \frac{d_{i}}{\beta^{i}}=\frac{\pi_{2}\left(K_{\beta}^{n}(\omega, x)\right)}{\beta^{n}} \rightarrow 0 \quad \text { as } n \rightarrow \infty .
$$

This shows that for all $\omega \in \Omega$ and for all $x \in[0,\lfloor\beta\rfloor /(\beta-1)]$ one has

$$
x=\sum_{i=1}^{\infty} \frac{d_{i}}{\beta^{i}}=\sum_{i=1}^{\infty} \frac{d_{i}(\omega, x)}{\beta^{i}} .
$$

The random procedure just described shows that to each $\omega \in \Omega$ corresponds an algorithm that produces expansions in base $\beta$. Further, if we identify the point $(\omega, x)$ with $\left(\omega,\left(d_{1}(\omega, x), d_{2}(\omega, x), \ldots\right)\right)$, then the action of $K_{\beta}$ on the second coordinate corresponds to the left shift.

In [DK2], the dynamical properties of the map $K_{\beta}$ were studied for $\beta$ satisfying $\beta^{2}=n \beta+k$ (with $1 \leq k \leq n$ ) and $\beta^{n}=\beta^{n-1}+\cdots+\beta+1$. It was shown that for these values of $\beta$, the underlying random $\beta$-transformation is isomorphic to a mixing Markov chain. However, the invariant measure considered in [DK2] is not the measure of maximal entropy (see Section 4, Remarks 6(3)). In this paper, we study the dynamical properties of $K_{\beta}$ for any non-integer $\beta>1$. In Section 2, we show that the map $K_{\beta}$ captures all possible expansions in base $\beta$ which are lexicographically ordered by the natural lexicographical ordering on $\Omega$. We also briefly discuss unique expansions. In Section 3 , we prove that the maximal entropy of $K_{\beta}$ is $\log (1+\lfloor\beta\rfloor)$. Further, $K_{\beta}$ has a unique measure $v_{\beta}$ of maximal entropy under which the random digits $\left(d_{i}\right)$, generated by the map $K_{\beta}$, form a uniform Bernoulli process. Moreover, the projection of the measure $v_{\beta}$ on the second coordinate is an infinite convolution of Bernoulli measures. In Section 4 , we show that if 1 has a finite greedy expansion of the form $1=b_{1} / \beta+b_{2} / \beta^{2}+\cdots+b_{n} / \beta^{n}$ with $b_{i} \geq 1$ for $i=1, \ldots, n$ and $n \geq 2$, then the measure $v_{\beta}$ is Markov, and the underlying Markov chain is explicitly given.

\section{Basic properties of random $\beta$-transformations}

Let $<_{\text {lex }}$ and $\leq_{\text {lex }}$ denote the lexicographical ordering on both $\Omega$ and $\{0,1, \ldots,\lfloor\beta\rfloor\}^{\mathbb{N}}$. For each $x \in[0,\lfloor\beta\rfloor /(\beta-1)]$, consider the set

$$
D_{x}=\left\{\left(d_{1}(\omega, x), d_{2}(\omega, x), \ldots\right): \omega \in \Omega\right\} .
$$

We now show that the elements of $D_{x}$ are ordered by the lexicographical ordering on $\Omega$. 
Theorem 1. Suppose $\omega, \omega^{\prime} \in \Omega$ are such that $\omega<_{\operatorname{lex}} \omega^{\prime}$. Then

$$
\left(d_{1}(\omega, x), d_{2}(\omega, x), \ldots\right) \leq_{\operatorname{lex}}\left(d_{1}\left(\omega^{\prime}, x\right), d_{2}\left(\omega^{\prime}, x\right), \ldots\right) .
$$

Proof. Let $i$ be the first index where $\omega$ and $\omega^{\prime}$ differ. Since $\omega<\operatorname{lex} \omega^{\prime}$, we have $\omega_{i}=0$ and $\omega_{i}^{\prime}=1$. Notice that $\pi_{2}\left(K_{\beta}^{j}(\omega, x)\right)=\pi_{2}\left(K_{\beta}^{j}\left(\omega^{\prime}, x\right)\right)$ for $j=0, \ldots, t_{i}$, where $t_{i} \geq 0$ is the time of the $i^{\text {th }}$ visit to the region $\Omega \times S$ of the orbit of $(\omega, x)$ under $K_{\beta}$. Then $d_{j}(\omega, x)=d_{j}\left(\omega^{\prime}, x\right)$ for all $j \leq t_{i}$.

If $t_{i}=\infty$, then $d_{j}(\omega, x)=d_{j}\left(\omega^{\prime}, x\right)$ for all $j$. If $t_{i}<\infty$, then $K_{\beta}^{t_{i}}(\omega, x)=$ $K_{\beta}^{t_{i}}\left(\omega^{\prime}, x\right) \in \Omega \times S$. Since $\omega_{i}=0$ and $\omega_{i}^{\prime}=1$, it follows that $d_{t_{i}+1}\left(\omega^{\prime}, x\right)=d_{t_{i}+1}(\omega, x)+1$. Hence,

$$
\left(d_{1}(\omega, x), d_{2}(\omega, x), \ldots\right)<_{\operatorname{lex}}\left(d_{1}\left(\omega^{\prime}, x\right), d_{2}\left(\omega^{\prime}, x\right), \ldots\right) .
$$

The next theorem shows that for all $x \in[0,\lfloor\beta\rfloor /(\beta-1)]$, any representation of $x$ of the form $x=\sum_{i=1}^{\infty} a_{i} / \beta^{i}$ with $a_{i} \in\{0,1, \ldots,\lfloor\beta\rfloor\}$ can be generated by means of the map $K_{\beta}$ by choosing an appropriate $\omega \in \Omega$.

Theorem 2. Let $x \in[0,\lfloor\beta\rfloor /(\beta-1)]$, and let $x=\sum_{i=1}^{\infty} a_{i} / \beta^{i}$ with $a_{i} \in\{0,1, \ldots,\lfloor\beta\rfloor\}$ be a representation of $x$ in base $\beta$. Then there exists an $\omega \in \Omega$ such that $a_{i}=d_{i}(\omega, x)$.

For the proof we need the following lemma.

Lemma 1. For $x \in[0,\lfloor\beta\rfloor /(\beta-1)]$, one has

(i) If $x \in E_{j}$ for some $j \in\{0, \ldots,\lfloor\beta\rfloor\}$, then $a_{1}=j$.

(ii) If $x \in S_{j}$ for some $j \in\{1, \ldots,\lfloor\beta\rfloor\}$, then $a_{1} \in\{j-1, j\}$.

Proof. The proof is by contradiction.

(i) Suppose $a_{1} \neq j$. If $a_{1} \leq j-1$, then $j \geq 1$ and

$$
x=\sum_{i=1}^{\infty} \frac{a_{i}}{\beta^{i}} \leq \frac{j-1}{\beta}+\sum_{i=2}^{\infty} \frac{\lfloor\beta\rfloor}{\beta^{i}}=\frac{j-1}{\beta}+\frac{\lfloor\beta\rfloor}{\beta(\beta-1)} .
$$

If $a_{1} \geq j+1$, then $j \leq\lfloor\beta\rfloor-1$ and $x \geq(j+1) / \beta$. In both cases $x \notin E_{j}$.

(ii) Suppose $a_{1} \notin\{j-1, j\}$. If $a_{1} \leq j-2$, then $j \geq 2$ and

$$
x \leq \frac{j-2}{\beta}+\frac{\lfloor\beta\rfloor}{\beta(\beta-1)} .
$$

If $a_{1} \geq j+1$, then $j \leq\lfloor\beta\rfloor-1$ and $x \geq(j+1) / \beta$. In both cases $x \notin S_{j}$.

Proof of Theorem 2 Define the numbers $\left\{x_{n}: n \in \mathbb{N}\right\}$ by $x_{n}=\sum_{i=1}^{\infty} a_{i+n-1} / \beta^{i}$. Notice that $x_{1}=x$. Furthermore, we define a set $\left\{\ell_{n}(x): n \in \mathbb{N}\right\}$ that keeps track of the number of times we flip a coin. More precisely,

$$
\ell_{n}(x)=\sum_{i=1}^{n} \mathbf{1}_{S}\left(x_{i}\right)
$$

We use induction on the number of digits already determined. 
- If $x \in E_{j}$, then $\ell_{1}(x)=0$ and by Lemma $1, a_{1}=j$. We set $\Omega_{1}=\Omega$.

- If $x \in S_{j}$, then $\ell_{1}(x)=1$ and by Lemma $1, a_{1} \in\{j-1, j\}$.

- If $a_{1}=j-1$, we set $\Omega_{1}=\left\{\omega \in \Omega: \omega_{1}=0\right\}$.

- If $a_{1}=j$, we set $\Omega_{1}=\left\{\omega \in \Omega: \omega_{1}=1\right\}$.

It follows that $\Omega_{1}$ is a cylinder of length $\ell_{1}(x)$, and $d_{1}(\omega, x)=a_{1}$ for all $\omega \in \Omega_{1}$. By a cylinder of length 0 we mean of course the whole space $\Omega$. Suppose we have obtained $\Omega_{n} \subseteq \cdots \subseteq \Omega_{1}$ so that $\Omega_{n}$ is a cylinder of length $\ell_{n}(x)$ and for all $\omega \in \Omega_{n}, d_{1}(\omega, x)=$ $a_{1}, \ldots, d_{n}(\omega, x)=a_{n}$. Notice that $x_{n+1}=\pi_{2}\left(K_{\beta}^{n}(\omega, x)\right)$ for all $\omega \in \Omega_{n}$.

- If $x_{n+1} \in E_{j}$, then $\ell_{n+1}(x)=\ell_{n}(x)$ and for all $\omega \in \Omega_{n}, d_{n+1}(\omega, x)=d_{1}\left(K_{\beta}^{n}(\omega, x)\right)$ $=j=a_{n+1}$, by Lemma 1 . We set $\Omega_{n+1}=\Omega_{n}$.

- If $x_{n+1} \in S_{j}$, then $\ell_{n+1}(x)=\ell_{n}(x)+1$ and $a_{n+1} \in\{j-1, j\}$ by Lemma 1 .

- If $a_{n+1}=j-1$, we set $\Omega_{n+1}=\left\{\omega \in \Omega_{n}: \omega_{\ell_{n+1}}=0\right\}$. Then, for all $\omega \in \Omega_{n+1}$, $d_{n+1}(\omega, x)=d_{1}\left(K_{\beta}^{n}(\omega, x)\right)=j-1=a_{n+1}$.

- If $a_{n+1}=j$, we set $\Omega_{n+1}=\left\{\omega \in \Omega_{n}: \omega_{\ell_{n+1}}=1\right\}$. Then, for all $\omega \in \Omega_{n+1}$, $d_{n+1}(\omega, x)=d_{1}\left(K_{\beta}^{n}(\omega, x)\right)=j=a_{n+1}$.

In all cases we see that $\Omega_{n+1}$ is a cylinder of length $\ell_{n+1}(x)$, and for all $\omega \in \Omega_{n+1}$, $d_{1}(\omega, x)=a_{1}, \ldots, d_{n+1}(\omega, x)=a_{n+1}$.

If the map $K_{\beta}$ hits the switch regions infinitely many times, then $\ell_{n}(x) \rightarrow \infty$ and, as is well known, $\bigcap \Omega_{n}$ consists of a single point. If this happens only finitely many times, then the set $\left\{\ell_{n}(x): n \in \mathbb{N}\right\}$ is finite and $\bigcap \Omega_{n}$ is exactly a cylinder set. In both cases $\bigcap \Omega_{n}$ is non-empty and $\omega \in \bigcap \Omega_{n}$ satisfies $d_{j}(\omega, x)=a_{j}$ for all $j \geq 1$.

Remark 1. Theorems 1 and 2 give another proof of the fact that among all possible $\beta$-expansions of a point $x \in[0,\lfloor\beta\rfloor /(\beta-1)]$, the greedy expansion is the largest in lexicographical order (it corresponds to the largest element $(1,1, \ldots)$ of $\Omega$ ), and the lazy one is the smallest (it corresponds to the smallest element $(0,0, \ldots)$ of $\Omega$ ). Furthermore, from Theorem 2, one sees that $x$ has a unique representation in base $\beta$ of the form

$$
x=\sum_{i=1}^{\infty} \frac{a_{i}}{\beta^{i}}
$$

with $a_{i} \in\{0,1, \ldots,\lfloor\beta\rfloor\}$ if and only if $a_{i}=d_{i}(\omega, x)$ for all $i \geq 1$ and all $\omega \in \Omega$. Equivalently, the greedy expansion of $x$ is the only representation of $x$ in base $\beta$ if and only if $x_{n} \in E$ for all $n \geq 1$. In this case, we have $x_{n}=T_{\beta}^{n-1} x=S_{\beta}^{n-1} x$ for all $n \geq 1$.

Remark 1 gives in fact a characterization of unique expansion in terms of the greedy expansion. Namely, if $x$ has an expansion of the form $x=a_{1} / \beta+a_{2} / \beta^{2}+\cdots$, then $x$ has a unique expansion in base $\beta$ if and only if $T_{\beta}^{n} x \in E_{a_{n+1}}$ for all $n \geq 0$. We would like to give other characterizations. Although some of the results are already known (see $[\overline{\mathrm{KL}}]$ ), we give simple proofs for completeness. We first observe that $1 \in S_{\lfloor\beta\rfloor} \cup E_{\lfloor\beta\rfloor}$, and $1 \in E_{\lfloor\beta\rfloor}$ if and only if $\lfloor\beta\rfloor /(\beta-1)-1 \in E_{0}$. The following proposition gives a characterization of the case $1 \in E_{\lfloor\beta\rfloor}$ using the greedy expansion of 1 . 
Proposition 1. Suppose 1 has a greedy expansion of the form $1=b_{1} / \beta+b_{2} / \beta^{2}+\cdots$.

(i) If $b_{i}=0$ for all $i \geq 3$, then $1 \in E_{b_{1}}$ if and only if $b_{2} \geq 2$. Moreover, if $b_{2}=1$, then $1=\lfloor\beta\rfloor /(\beta-1)-1 / \beta$.

(ii) If $b_{i} \geq 1$ for some $i \geq 3$, then $1 \in E_{b_{1}}$ if and only if $b_{2} \geq 1$.

Proof. First observe that $\lfloor\beta\rfloor=b_{1}$, and that

$$
1=\frac{b_{1}}{\beta}+\frac{b_{2}}{\beta^{2}}+\frac{1}{\beta^{2}} T_{\beta}^{2} 1 .
$$

This implies that $\beta^{2}-b_{1} \beta=b_{2}+T_{\beta}^{2} 1$. Now, by definition $1 \in E_{b_{1}}$ if and only if $1>b_{1} /(\beta-1)-1 / \beta$, or equivalently $\beta^{2}-b_{1} \beta>1$.

In case (i), we have $T_{\beta}^{2} 1=0$, which implies that $\beta^{2}-b_{1} \beta=b_{2}$. Hence, $1 \in E_{b_{1}}$ if and only if $b_{2} \geq 2$. If $b_{2}=1$, then $\beta^{2}-b_{1} \beta=1$; equivalently, $1=\lfloor\beta\rfloor /(\beta-1)-1 / \beta$.

In case (ii), we have $0<T_{\beta}^{2} 1<1$. Hence, $\beta^{2}-b_{1} \beta=b_{2}+T_{\beta}^{2} 1>1$ if and only if $b_{2} \geq 1$.

Before we proceed to the characterization of the uniqueness of the $\beta$-expansion of $x$, we need the following simple lemma.

Lemma 2. Suppose $x$ has a greedy expansion of the form $x=a_{1} / \beta+a_{2} / \beta^{2}+\cdots$. If $a_{n+1} \geq 1$, then $T_{\beta}^{n} x \in E_{a_{n+1}}$ if and only if $T_{\beta}^{n+1} x>\lfloor\beta\rfloor /(\beta-1)-1$.

Proof. Notice that

$$
T_{\beta}^{n} x=\frac{a_{n+1}}{\beta}+\frac{1}{\beta} T_{\beta}^{n+1} x \in S_{a_{n+1}} \cup E_{a_{n+1}} .
$$

Thus, $T_{\beta}^{n} x \in E_{a_{n+1}}$ if and only if

$$
T_{\beta}^{n} x>\frac{\lfloor\beta\rfloor}{\beta(\beta-1)}+\frac{a_{n+1}-1}{\beta} .
$$

Rewriting one finds that $T_{\beta}^{n} x \in E_{a_{n+1}}$ if and only if $T_{\beta}^{n+1} x>\lfloor\beta\rfloor /(\beta-1)-1$.

Note that if $a_{n+1}=0$, then $T_{\beta}^{n} x \in E_{0}$.

The following theorem is an immediate consequence of the above lemma. We remark that a lexicographical version of this theorem was obtained independently for the case $x=1$, and via other methods in [KL, Theorem 3.1].

Theorem 3. Suppose $x$ has a greedy expansion of the form $x=a_{1} / \beta+a_{2} / \beta^{2}+\cdots$. Then $x$ has a unique expansion in base $\beta$ if and only if $T_{\beta}^{n+1} x>\lfloor\beta\rfloor /(\beta-1)-1$ for all $n \geq 0$ with $a_{n+1} \geq 1$.

Corollary 1. Suppose $x$ has a greedy expansion of the form $x=a_{1} / \beta+a_{2} / \beta^{2}+\cdots$ with $a_{i} \geq 1$ for all $i \geq 1$. Then $x$ has a unique $\beta$-expansion.

Proof. Observe that $T_{\beta}^{n} x \geq 1 /(\beta-1)$ for all $n \geq 0$, and $1 /(\beta-1)>\lfloor\beta\rfloor /(\beta-1)-1$. The result follows from Theorem 3 . 
Corollary 2. If 1 has a unique $\beta$-expansion, then there exists a $k \geq 1$ such that in the greedy expansion of 1 , every block of consecutive zeros consists of at most $k$ terms.

Proof. Let $1=b_{1} / \beta+b_{2} / \beta^{2}+\cdots$ be the greedy expansion. By uniqueness $1 \in E_{b_{1}}$, so $b_{1} /(\beta-1)-1<1 / \beta$. Hence, there exists a $k \geq 1$ such that

$$
\frac{1}{\beta^{k+1}} \leq \frac{b_{1}}{\beta-1}-1<\frac{1}{\beta^{k}}
$$

If $b_{i-1} b_{i} \ldots b_{j}$ is a block with $b_{i-1} \geq 1, b_{i}=\cdots=b_{j}=0$ and $j-i+1 \geq k+1$, then

$$
T_{\beta}^{i-1} 1<\frac{1}{\beta^{k+1}} \leq \frac{b_{1}}{\beta-1}-1,
$$

contradicting Theorem 3

Another immediate corollary of Theorem 3 and Proposition 1 is the following.

Corollary 3. Suppose 1 has an infinite greedy expansion of the form $1=b_{1} / \beta+b_{2} / \beta^{2}$ $+\cdots$ with $b_{2} \geq 1$. Let $k \geq 1$ be the unique integer such that

$$
\frac{1}{\beta^{k+1}} \leq \frac{b_{1}}{\beta-1}-1<\frac{1}{\beta^{k}} .
$$

If in the greedy expansion of 1 every block of consecutive zeros contains at most $k-1$ terms, then 1 has a unique $\beta$-expansion.

\section{Measures of maximal entropy for random $\beta$-expansions}

In this section we show that the map $K_{\beta}$ on $\Omega \times[0,\lfloor\beta\rfloor /(\beta-1)]$ can be essentially identified with the left shift on $\{0, \ldots,\lfloor\beta\rfloor\}^{\mathbb{N}}$. This will enable us to prove that $K_{\beta}$ has a unique measure of maximal entropy.

Let $D=\{0, \ldots,\lfloor\beta\rfloor\}^{\mathbb{N}}$ be equipped with the product $\sigma$-algebra $\mathcal{D}$ and the uniform product measure $\mathbb{P}$. Let $\sigma^{\prime}$ be the left shift on $D$. On the set $\Omega \times[0,\lfloor\beta\rfloor /(\beta-1)]$ we consider the product $\sigma$-algebra $\mathcal{A} \times \mathcal{B}$, where $\mathcal{B}$ is the Borel $\sigma$-algebra on $[0,\lfloor\beta\rfloor /(\beta-1)]$, and $\mathcal{A}$ the product $\sigma$-algebra on $\Omega$. Define the function $\varphi: \Omega \times[0,\lfloor\beta\rfloor /(\beta-1)] \rightarrow D$ by

$$
\varphi(\omega, x)=\left(d_{1}(\omega, x), d_{2}(\omega, x), \ldots\right) .
$$

It is easily seen that $\varphi$ is measurable, and $\varphi \circ K_{\beta}=\sigma^{\prime} \circ \varphi$. Furthermore, Theorem 2 implies that $\varphi$ is surjective. We will now show that $\varphi$ restricted to an appropriate $K_{\beta^{-}}$ invariant subset is in fact invertible. Let

$$
\begin{aligned}
Z & =\left\{(\omega, x) \in \Omega \times[0,\lfloor\beta\rfloor /(\beta-1)]: K_{\beta}^{n}(\omega, x) \in \Omega \times S \text { infinitely often }\right\}, \\
D^{\prime} & =\left\{\left(a_{1}, a_{2}, \ldots\right) \in D: \sum_{i=1}^{\infty} \frac{a_{j+i-1}}{\beta^{i}} \in S \text { for infinitely many } j \text { 's }\right\} .
\end{aligned}
$$

Then $\varphi(Z)=D^{\prime}, K_{\beta}^{-1}(Z)=Z$ and $\left(\sigma^{\prime}\right)^{-1}\left(D^{\prime}\right)=D^{\prime}$. Let $\varphi^{\prime}$ be the restriction of the $\operatorname{map} \varphi$ to $Z$. 
Lemma 3. The map $\varphi^{\prime}: Z \rightarrow D^{\prime}$ is a bimeasurable bijection.

Proof. For any sequence $\left(a_{1}, a_{2}, \ldots\right) \in D^{\prime}$, define recursively

$$
r_{1}=\min \left\{j \geq 1: \sum_{l=1}^{\infty} \frac{a_{j+l-1}}{\beta^{l}} \in S\right\}, \quad r_{i}=\min \left\{j>r_{i-1}: \sum_{l=1}^{\infty} \frac{a_{j+l-1}}{\beta^{l}} \in S\right\} .
$$

If $\sum_{l=1}^{\infty} a_{r_{i}+l-1} / \beta^{l} \in S_{j}$ then, according to Lemma $1, a_{r_{i}} \in\{j-1, j\}$. If $a_{r_{i}}=j-1$, let $\omega_{i}=0$, otherwise let $\omega_{i}=1$. Define $\left(\varphi^{\prime}\right)^{-1}: D^{\prime} \rightarrow Z$ by

$$
\left(\varphi^{\prime}\right)^{-1}\left(\left(a_{1}, a_{2}, \ldots\right)\right)=\left(\omega, \sum_{i=1}^{\infty} \frac{a_{i}}{\beta^{i}}\right) .
$$

It is easily checked that $\left(\varphi^{\prime}\right)^{-1}$ is measurable, and is the inverse of $\varphi^{\prime}$.

Lemma 4. $\mathbb{P}\left(D^{\prime}\right)=1$.

Proof. For any sequence $\left(a_{1}, a_{2}, \ldots\right) \in D$ and $m \geq 1$, define

$$
x_{m}=\frac{1}{\beta}+\frac{a_{1}}{\beta^{m+1}}+\frac{a_{2}}{\beta^{m+2}}+\cdots .
$$

Clearly $x_{m} \geq 1 / \beta$. On the other hand,

$$
x_{m} \leq \frac{1}{\beta}+\sum_{i=1}^{\infty} \frac{\lfloor\beta\rfloor}{\beta^{m+i}}=\frac{1}{\beta}\left(1+\frac{\lfloor\beta\rfloor}{\beta^{m-1}(\beta-1)}\right) .
$$

Since $1+\frac{\lfloor\beta\rfloor}{\beta^{m-1}(\beta-1)} \downarrow 1$ as $m \rightarrow \infty$, there exists an integer $N>0$ such that for all $m \geq N$,

$$
\frac{1}{\beta} \leq x_{m} \leq \frac{\lfloor\beta\rfloor}{\beta(\beta-1)},
$$

i.e. $x_{m} \in S_{1}$ for all $m \geq N$. Let

$$
D^{\prime \prime}=\{\left(a_{1}, a_{2}, \ldots\right) \in D: a_{j} a_{j+1} \ldots a_{j+N-1}=\underbrace{00 \ldots 0}_{N-1 \text { zeros }} \text { for infinitely many } j\} .
$$

From the above, we conclude that $D^{\prime \prime} \subseteq D^{\prime}$. Clearly $\mathbb{P}\left(D^{\prime \prime}\right)=1$, hence $\mathbb{P}\left(D^{\prime}\right)=1$.

Now, consider the $K_{\beta}$-invariant measure $v_{\beta}$ defined on $\mathcal{A} \times \mathcal{B}$ by $v_{\beta}(A)=\mathbb{P}(\varphi(Z \cap A))$. The following theorem is a simple consequence of Lemmas 3 and 4

Theorem 4. Let $\beta>1$ be a non-integer. The dynamical systems $(\Omega \times[0,\lfloor\beta\rfloor /(\beta-1)]$, $\left.\mathcal{A} \times \mathcal{B}, v_{\beta}, K_{\beta}\right)$ and $\left(D, \mathcal{D}, \mathbb{P}, \sigma^{\prime}\right)$ are measurably isomorphic.

Remark 2. The above theorem implies that $h_{v_{\beta}}\left(K_{\beta}\right)=\log (1+\lfloor\beta\rfloor)$. Further, since $\mathbb{P}$ is the unique measure of maximal entropy on $D$, we see that $\nu_{\beta}$ is the only $K_{\beta}$-invariant measure with support $Z$ and maximal entropy $\log (1+\lfloor\beta\rfloor)$, i.e. any other $K_{\beta}$-invariant measure with support $Z$ has entropy strictly less than $\log (1+\lfloor\beta\rfloor)$. We now investigate the entropy of $K_{\beta}$-invariant measures $\mu$ for which $\mu\left(Z^{c}\right)>0$. 
Lemma 5. Let $\mu$ be a $K_{\beta}$-invariant measure for which $\mu\left(Z^{c}\right)>0$. Then $h_{\mu}\left(K_{\beta}\right)<$ $\log (1+\lfloor\beta\rfloor)$.

Proof. Since $Z$ and $Z^{c}$ are $K_{\beta}$-invariant, there exist $0 \leq \alpha<1$ and $K_{\beta}$-invariant measures $\mu_{1}, \mu_{2}$ concentrated on $Z$ and $Z^{c}$ respectively, such that $\mu=\alpha \mu_{1}+(1-\alpha) \mu_{2}$. Then $h_{\mu}\left(K_{\beta}\right)=\alpha h_{\mu_{1}}\left(K_{\beta}\right)+(1-\alpha) h_{\mu_{2}}\left(K_{\beta}\right)$. From Remark 2 we have $h_{\mu_{1}}\left(K_{\beta}\right) \leq$ $\log (1+\lfloor\beta\rfloor)$. We now show that $h_{\mu_{2}}\left(K_{\beta}\right)<\log (1+\lfloor\beta\rfloor)$. To this end, let

$$
G=\{x \in[0,\lfloor\beta\rfloor /(\beta-1)]: x \text { has a unique } \beta \text {-expansion }\} .
$$

Then $\Omega \times G \subseteq K_{\beta}^{-1}(\Omega \times G)$, and $\bigcup_{i=0}^{\infty} K_{\beta}^{-i}(\Omega \times G)=Z^{c}$. From the above we see that $\mu_{2}(\Omega \times G)=1$, hence it is enough to study the entropy of the map $K_{\beta}$ restricted to $\Omega \times G$. On this set $K_{\beta}$ has the form $I_{\Omega} \times T_{\beta}$, where $I_{\Omega}$ is the identity map on $\Omega$, and $T_{\beta}$ the greedy map restricted to $G$. On $G$ we consider the Borel $\sigma$-algebra $G \cap \mathcal{B}$. Notice that $\mu_{2} \circ \pi_{2}^{-1}$ is a $T_{\beta}$-invariant measure with support $G$, hence $h_{\mu_{2} \circ \pi_{2}^{-1}}\left(T_{\beta}\right) \leq \log \beta$.

Let $\mathcal{F}$ and $\mathcal{G}$ be any two measurable partitions of $\Omega$ and $G$ respectively. For any $n \geq 1$,

$$
\bigvee_{i=0}^{n-1} K_{\beta}^{-i}(\mathcal{F} \times \mathcal{G})=\bigvee_{i=0}^{n-1}\left(I_{\Omega} \times T_{\beta}\right)^{-i}(\mathcal{F} \times \mathcal{G})=\mathcal{F} \times \bigvee_{i=0}^{n-1} T_{\beta}^{-i} \mathcal{G}
$$

modulo sets of $\mu_{2}$-measure 0 . Hence,

$$
\begin{aligned}
H_{\mu_{2}}\left(\Omega \times \bigvee_{i=0}^{n-1} T_{\beta}^{-i} \mathcal{G}\right) & \leq H_{\mu_{2}}\left(\bigvee_{i=0}^{n-1} K_{\beta}^{-i}(\mathcal{F} \times \mathcal{G})\right) \\
& \leq H_{\mu_{2}}(\mathcal{F} \times G)+H_{\mu_{2}}\left(\Omega \times \bigvee_{i=0}^{n-1} T_{\beta}^{-i} \mathcal{G}\right)
\end{aligned}
$$

Now, dividing by $n$ and taking the limit as $n \rightarrow \infty$, we get

$$
h_{\mu_{2}}\left(K_{\beta}, \mathcal{F} \times \mathcal{G}\right)=h_{\mu_{2}}\left(K_{\beta}, \Omega \times \mathcal{G}\right)=h_{\mu_{2} \circ \pi_{2}^{-1}}\left(T_{\beta}, \mathcal{G}\right) \leq \log \beta .
$$

Since $\mathcal{F}$ and $\mathcal{G}$ are arbitrary partitions, we have

$$
h_{\mu_{2}}\left(K_{\beta}\right) \leq \log \beta<\log (1+\lfloor\beta\rfloor) .
$$

Therefore, $h_{\mu}\left(K_{\beta}\right)<\log (1+\lfloor\beta\rfloor)$.

From Remark 2 and Lemma 5 we arrive at the following theorem.

Theorem 5. The measure $v_{\beta}$ is the unique $K_{\beta}$-invariant measure of maximal entropy.

An interesting consequence of the above theorems is that if $\beta, \beta^{\prime}>1$ are non-integers, then

$$
\lfloor\beta\rfloor=\left\lfloor\beta^{\prime}\right\rfloor \quad \text { if and only if } \quad\left(K_{\beta}, v_{\beta}\right) \text { is isomorphic to }\left(K_{\beta^{\prime}}, v_{\beta^{\prime}}\right) \text {. }
$$

As before, let $\pi_{2}: \Omega \times[0,\lfloor\beta\rfloor /(\beta-1)] \rightarrow[0,\lfloor\beta\rfloor /(\beta-1)]$ be the natural projection $\pi_{2}(\omega, x)=x$. We are interested in identifying the projection of the measure $v_{\beta}$ on the 
second coordinate, that is, the measure $v_{\beta} \circ \pi_{2}^{-1}$ defined on $[0,\lfloor\beta\rfloor /(\beta-1)]$. To do that, we consider the purely discrete measures $\left\{\delta_{i}\right\}_{i \geq 1}$ defined on $\mathbb{R}$ as follows:

$$
\delta_{i}(\{0\})=\frac{1}{\lfloor\beta\rfloor+1}, \quad \ldots, \quad \delta_{i}\left(\left\{\lfloor\beta\rfloor \beta^{-i}\right\}\right)=\frac{1}{\lfloor\beta\rfloor+1} .
$$

Let $\delta$ be the corresponding infinite Bernoulli convolution,

$$
\delta=\lim _{n \rightarrow \infty} \delta_{1} * \ldots * \delta_{n}
$$

Theorem 6. $v_{\beta} \circ \pi_{2}^{-1}=\delta$.

Proof. Let $h: D \rightarrow[0,\lfloor\beta\rfloor /(\beta-1)]$ be given by $h(y)=\sum_{i=1}^{\infty} y_{i} / \beta^{i}$, where $y=$ $\left(y_{1}, y_{2}, \ldots\right)$. Then $\pi_{2}=h \circ \varphi$ and $\delta=\mathbb{P} \circ h^{-1}$. Since $\mathbb{P}=v_{\beta} \circ \varphi^{-1}$, it follows that $v_{\beta} \circ \pi_{2}^{-1}=\delta$.

If $\beta \in(1,2)$ then $\delta$ is an Erdős measure on $[0,1 /(\beta-1)]$, and lots of things are already known. For example, if $\beta$ is a Pisot number, then $\delta$ is singular with respect to Lebesgue measure $\lambda$ ([E1], [E2], [S]). Further, for almost all $\beta \in(1,2)$ the measure $\delta$ is equivalent to $\lambda$ ([So], [MS]). There are many generalizations of these results to the case of an arbitrary digit set (see [PSS] for more references and results).

\section{Finite greedy expansion of 1 with positive coefficients, and the Markov property of the random $\beta$-expansion}

We now assume that the greedy expansion of 1 in base $\beta$ satisfies $1=b_{1} / \beta+b_{2} / \beta^{2}+$ $\cdots+b_{n} / \beta^{n}$ with $b_{i} \geq 1$ for $i=1, \ldots, n$ and $n \geq 2$ (notice that $\lfloor\beta\rfloor=b_{1}$ ). We show that in this case the dynamics of $K_{\beta}$ can be identified with a subshift of finite type with an irreducible adjacency matrix. As a result the unique measure of maximal entropy $v_{\beta}$ obtained in the previous section is Markov.

The analysis of the case $\beta^{2}=b_{1} \beta+1$ needs some adjustments. For this reason, we assume here that $\beta^{2} \neq b_{1} \beta+1$, and refer the reader to the end of this section (Remarks $6(2))$ for the appropriate modifications needed for the case $\beta^{2}=b_{1} \beta+1$.

We begin by a proposition that is an immediate consequence of Proposition 1 and Lemma 2, and which plays a crucial role in finding the Markov partition describing the dynamics of the map $K_{\beta}$, as defined in Section 1 .

Proposition 2. Suppose 1 has a finite greedy expansion of the form $1=b_{1} / \beta+b_{2} / \beta^{2}+$ $\cdots+b_{n} / \beta^{n}$. If $b_{j} \geq 1$ for $1 \leq j \leq n$, then

(i) $T_{\beta}^{i} 1=S_{\beta}^{i} 1 \in E_{b_{i+1}}, 0 \leq i \leq n-2$.

(ii) $T_{\beta}^{n-1} 1=S_{\beta}^{n-1} 1=b_{n} / \beta \in S_{b_{n}}, T_{\beta}^{n} 1=0$, and $S_{\beta}^{n} 1=1$.

(iii) $T_{\beta}^{i}\left(\frac{b_{1}}{\beta-1}-1\right)=S_{\beta}^{i}\left(\frac{b_{1}}{\beta-1}-1\right) \in E_{b_{1}-b_{i+1}}, 0 \leq i \leq n-2$. 
(iv) $T_{\beta}^{n-1}\left(\frac{b_{1}}{\beta-1}-1\right)=S_{\beta}^{n-1}\left(\frac{b_{1}}{\beta-1}-1\right)=\frac{b_{1}}{\beta(\beta-1)}+\frac{b_{1}-b_{n}}{\beta} \in S_{b_{1}-b_{n}+1}$, $T_{\beta}^{n}\left(\frac{b_{1}}{\beta-1}-1\right)=\frac{b_{1}}{\beta-1}-1, \quad S_{\beta}^{n}\left(\frac{b_{1}}{\beta-1}-1\right)=\frac{b_{1}}{\beta-1}$.

Moreover, by Proposition 1 and Lemma 2, one has

$$
\begin{aligned}
T_{\beta}^{i} 1 & =S_{\beta}^{i} 1>\frac{b_{1}}{\beta-1}-1, \\
T_{\beta}^{i}\left(\frac{b_{1}}{\beta-1}-1\right) & =S_{\beta}^{i}\left(\frac{b_{1}}{\beta-1}-1\right)<1 \quad \text { for all } i=1, \ldots, n-1 .
\end{aligned}
$$

To find the Markov chain behind the map $K_{\beta}$, one starts by refining the partition

$$
\mathcal{E}=\left\{E_{0}, S_{1}, E_{1}, \ldots, S_{b_{1}}, E_{b_{1}}\right\}
$$

of $\left[0, b_{1} /(\beta-1)\right]$, using the orbits of 1 and $b_{1} /(\beta-1)-1$ under the transformation $T_{\beta}$. We place the endpoints of $\mathcal{E}$ together with $T_{\beta}^{i} 1, T_{\beta}^{i}\left(b_{1} /(\beta-1)-1\right), i=0, \ldots, n-2$, in increasing order. We use these points to form a new partition $\mathcal{C}$ which is a refinement of $\mathcal{E}$, consisting of intervals. We write $\mathcal{C}$ as

$$
\mathcal{C}=\left\{C_{0}, C_{1}, \ldots, C_{L}\right\}
$$

We choose $\mathcal{C}$ to satisfy the following. For $0 \leq i \leq n-2$,

- $T_{\beta}^{i} 1 \in C_{j}$ if and only if $T_{\beta}^{i} 1$ is a left endpoint of $C_{j}$,

$-T_{\beta}^{i}\left(b_{1} /(\beta-1)-1\right) \in C_{j}$ if and only if $T_{\beta}^{i}\left(b_{1} /(\beta-1)-1\right)$ is a right endpoint of $C_{j}$.

Notice that this choice is possible, since the points $T_{\beta}^{i} 1, T_{\beta}^{i}\left(b_{1} /(\beta-1)-1\right)$ for $0 \leq i \leq$ $n-2$ are all different.

Recall that the map $\ell:[0,\lfloor\beta\rfloor /(\beta-1)] \rightarrow[0,\lfloor\beta\rfloor /(\beta-1)]$ defined by $\ell(x)=$ $\lfloor\beta\rfloor /(\beta-1)-x$ satisfies $T_{\beta} \circ \ell=\ell \circ S_{\beta}$. Thus, if $x \in E_{i}$ for some $i$, then $T_{\beta} x=S_{\beta} x$ and $T_{\beta} \ell(x)=\ell T_{\beta}(x)$. From the dynamics of $K_{\beta}$ on this refinement, one reads the following properties of $\mathcal{C}$.

p1. $C_{0}=\left[0, b_{1} /(\beta-1)-1\right]$ and $C_{L}=\left[1, b_{1} /(\beta-1)\right]$.

p2. For $i=0,1, \ldots, b_{1}, E_{i}$ can be written as a finite disjoint union $\bigcup_{j \in M_{i}} C_{j}$ with $M_{0}, M_{1}, \ldots, M_{b_{1}}$ disjoint subsets of $\{0,1, \ldots, L\}$. Further, the number of elements in $M_{i}$ equals the number of elements in $M_{b_{1}-i}$.

p3. To each $S_{i}$ there corresponds exactly one $j \in\{0,1, \ldots, L\} \backslash \bigcup_{k=0}^{b_{1}} M_{k}$ such that $S_{i}=C_{j}$. This is possible since the $T_{\beta}$-orbits of 1 and $b_{1} /(\beta-1)-1$ never hit the interior of $\bigcup_{i=1}^{b_{1}} S_{i}$.

p4. If $C_{j} \subset E_{i}$, then $T_{\beta}\left(C_{j}\right)=S_{\beta}\left(C_{j}\right)$ is a finite disjoint union of elements of $\mathcal{C}$, say $T_{\beta}\left(C_{j}\right)=C_{i_{1}} \cup \cdots \cup C_{i_{l}}$. Since $\ell\left(C_{j}\right)=C_{L-j} \subset E_{b_{1}-i}$, it follows that $T_{\beta}\left(C_{L-j}\right)=$ $C_{L-i_{1}} \cup \cdots \cup C_{L-i_{l}}$.

p5. If $C_{j}=S_{i}$, then $T_{\beta}\left(C_{j}\right)=C_{0}$ and $S_{\beta}\left(C_{j}\right)=C_{L}$. 
Define the partition $\mathcal{P}$ of $\Omega \times\left[0, b_{1} /(\beta-1)\right]$ by

$$
\mathcal{P}=\left\{\Omega \times C_{j}: j \in \bigcup_{k=0}^{b_{1}} M_{k}\right\} \cup\left\{\left\{\omega_{1}=i\right\} \times S_{j}: i=0,1, j=1, \ldots, b_{1}\right\} .
$$

From $\mathbf{p} 4$ and $\mathbf{p 5}$ we conclude that $\mathcal{P}$ is a Markov partition underlying the map $K_{\beta}$.

To define the underlying subshift of finite type associated with $K_{\beta}$, we consider the $(L+1) \times(L+1)$ matrix $A=\left(a_{i, j}\right)$ with entries in $\{0,1\}$ defined by

$$
a_{i, j}= \begin{cases}1 & \text { if } i \in \bigcup_{k=0}^{b_{1}} M_{k} \text { and } \lambda\left(C_{j} \cap T_{\beta}\left(C_{i}\right)\right)=\lambda\left(C_{j}\right), \\ 0 & \text { if } i \in \bigcup_{k=0}^{b_{1}} M_{k} \text { and } C_{i} \cap T_{\beta}^{-1} C_{j}=\emptyset \\ 1 & \text { if } i \in\{0, \ldots, L\} \backslash \bigcup_{k=0}^{b_{1}} M_{k} \text { and } j=0, L \\ 0 & \text { if } i \in\{0, \ldots, L\} \backslash \bigcup_{k=0}^{b_{1}} M_{k} \text { and } j \neq 0, L\end{cases}
$$

Remark 3. Because of our assumption $\beta^{2} \neq b_{1} \beta+1$, we have $\lambda\left(C_{j} \cap T_{\beta}\left(C_{i}\right)\right)=\lambda\left(C_{j}\right)$ if and only if $C_{j} \subseteq T_{\beta}\left(C_{i}\right)$. However, for the analysis of the case $\beta^{2}=b_{1} \beta+1$, we need the definition of the matrix $A$ as given in equation (1).

Let $Y$ denote the topological Markov chain (or the subshift of finite type) determined by the matrix $A$, that is, $Y=\left\{y=\left(y_{i}\right) \in\{0,1, \ldots, L\}^{\mathbb{N}}: a_{y_{i} y_{i+1}}=1\right\}$. We let $\sigma_{Y}$ be the left shift on $Y$. For ease of notation, we denote by $s_{1}, \ldots, s_{b_{1}}$ the states $j \in\{0, \ldots, L\} \backslash$ $\bigcup_{k=0}^{b_{1}} M_{k}$ corresponding to the switch regions $S_{1}, \ldots, S_{b_{1}}$ respectively.

To each $y \in Y$, we associate a sequence $\left(e_{i}\right) \in\left\{0,1, \ldots, b_{1}\right\}^{\mathbb{N}}$ and a point $x \in$ $\left[0, b_{1} /(\beta-1)\right]$ as follows. Let

$$
e_{j}= \begin{cases}i & \text { if } y_{j} \in M_{i} \\ i & \text { if } y_{j}=s_{i} \text { and } y_{j+1}=0 \\ i-1 & \text { if } y_{j}=s_{i} \text { and } y_{j+1}=L\end{cases}
$$

Now set

$$
x=\sum_{j=1}^{\infty} \frac{e_{j}}{\beta^{j}} .
$$

Our aim is to define a map $\psi: Y \rightarrow \Omega \times\left[0, b_{1} /(\beta-1)\right]$ that intertwines the actions of $K_{\beta}$ and $\sigma_{Y}$. Given $y \in Y$, equations $(2)$ and (3) describe what the second coordinate of $\psi$ should be. In order to be able to associate an $\omega \in \Omega$, one needs that $y_{i} \in\left\{s_{1}, \ldots s_{b_{1}}\right\}$ infinitely often. For this reason it is not possible to define $\psi$ on all of $Y$, but only on an invariant subset. To be more precise, let

$$
Y^{\prime}=\left\{y=\left(y_{1}, y_{2}, \ldots\right) \in Y: y_{i} \in\left\{s_{1}, \ldots, s_{b_{1}}\right\} \text { for infinitely many } i \text { 's }\right\} .
$$

Define $\psi: Y^{\prime} \rightarrow \Omega \times\left[0, b_{1} /(\beta-1)\right]$ as follows. Let $y=\left(y_{1}, y_{2}, \ldots\right) \in Y^{\prime}$, and define $x$ as in (3). To define a point $\omega \in \Omega$ corresponding to $y$, we first locate the indices $n_{i}=$ $n_{i}(y)$ where the realization $y$ of the Markov chain is in state $s_{r}$ for some $r \in\left\{1, \ldots, b_{1}\right\}$. 
That is, let $n_{1}<n_{2}<\cdots$ be the indices such that $y_{n_{i}}=s_{r}$ for some $r=1, \ldots, b_{1}$. Define

$$
\omega_{j}= \begin{cases}1 & \text { if } y_{n_{j}+1}=0 \\ 0 & \text { if } y_{n_{j}+1}=L .\end{cases}
$$

Now set $\psi(y)=(\omega, x)$.

The following two lemmas reflect the fact that the dynamics of $K_{\beta}$ is essentially the same as that of the Markov chain $Y$. These lemmas are generalizations of Lemmas 1 and 2 in [DK2], and the proofs are slight modifications of the arguments there.

Lemma 6. Let $y \in Y^{\prime}$ be such that $\psi(y)=(\omega, x)$. Then

(i) $y_{1}=k$ for some $k \in \bigcup_{i=0}^{b_{1}} M_{i} \Rightarrow x \in C_{k}$.

(ii) $y_{1}=s_{i}, y_{2}=0 \Rightarrow x \in S_{i}$ and $\omega_{1}=1$ for $i=1, \ldots, b_{1}$.

(iii) $y_{1}=s_{i}, y_{2}=L \Rightarrow x \in S_{i}$ and $\omega_{1}=0$ for $i=1, \ldots, b_{1}$.

Lemma 7. For $y \in Y^{\prime}$, we have

$$
\psi \circ \sigma_{Y}(y)=K_{\beta} \circ \psi(y) .
$$

Remark 4. From Lemmas 6 and 7 we have the following. If $y \in Y^{\prime}$ with $\psi(y)=(\omega, x)$, then for any $i \geq 1$ and any $k \in\{0,1, \ldots, L\}$,

$$
y_{i}=k \Rightarrow \pi_{2}\left(K_{\beta}^{i-1}(\omega, x)\right) \in C_{k} .
$$

Having defined the map $\psi$ with the above properties, we now consider the measure $Q$ of maximal entropy on $Y$. This measure is unique since the adjacency matrix $A=\left(a_{i, j}\right)$, as defined in [1], is irreducible [W] Theorem 8.10]. In order to describe $Q$ explicitly, we first study the matrix $A$. From the dynamics of $K_{\beta}$ as well as properties p1-p5 one easily sees that $A$ has the following properties:

(i) $a_{i, j}=a_{L-i, L-j}$ for all $i, j=0,1, \ldots, L$,

(ii) $\sum_{i=0}^{L} a_{i, j}=b_{1}+1$ for all $j=0,1, \ldots, L$.

By induction one can easily show that if $A^{k}=\left(a_{i, j}^{(k)}\right)$, then $A^{k}$ satisfies

(iii) $a_{i, j}^{(k)}=a_{L-i, L-j}^{(k)}$ for all $i, j=0,1, \ldots, L$,

(iv) $\sum_{i=0}^{L} a_{i, j}^{(k)}=\left(b_{1}+1\right)^{k}$ for all $j=0,1, \ldots, L$.

Since $A$ is an irreducible, non-negative integral matrix, we calculate the topological entropy $h(Y)$ of $Y$ by the formula

$$
h(Y)=\lim _{n \rightarrow \infty} \frac{1}{n} \log \left|B_{n}(Y)\right|,
$$

where $B_{n}(Y)$ denotes the collection of blocks of length $n$ in the shift space $Y$. According to property (iv) above $\left|B_{n}(Y)\right|=\sum_{i, j} a_{i, j}^{(n)}=(L+1)\left(b_{1}+1\right)^{n}$. Hence $h(Y)=\log \left(b_{1}+1\right)$. It follows that the Perron eigenvalue $\lambda_{A}$ equals $b_{1}+1$ (i.e. the largest positive eigenvalue of the matrix $A$ ). To determine the measure of maximal entropy we need to find a positive 
left eigenvector $u$ and a positive right eigenvector $v$. According to property (ii) above a left eigenvector is given by $u=(1,1, \ldots, 1)$. For the positive right eigenvector $v$, we choose $v$ to satisfy $\sum_{i=0}^{L} v_{i}=1$. Using the technique developed by Parry, we show that the measure $Q$ of maximal entropy is the Markov measure generated by the transition matrix $P=\left(p_{i, j}\right)$, where $p_{i, j}=a_{i, j} \frac{v_{j}}{\left(b_{1}+1\right) v_{i}}$, and stationary distribution $p=v$. We equip the space $Y$ with the $\sigma$-algebra $\mathcal{H}$ generated by the cylinders. We have the following theorem.

Theorem 7. The dynamical systems $\left(\Omega \times\left[0, b_{1} /(\beta-1)\right], \mathcal{A} \times \mathcal{B}, Q \circ \psi^{-1}, K_{\beta}\right)$ and $\left(Y, \mathcal{H}, Q, \sigma_{Y}\right)$ are measurably isomorphic.

Proof. We show that the map $\psi: Y^{\prime} \rightarrow Z$ is the required isomorphism. From Lemma 7 we find that $\psi$ intertwines the actions of $K_{\beta}$ and $\sigma_{Y}$. Furthermore, it is easily checked that $\psi: Y^{\prime} \rightarrow Z$ is a bimeasurable bijection. The inverse $\psi^{-1}: Z \rightarrow Y^{\prime}$ is given by $\psi^{-1}(\omega, x)=y$, where $y_{i}=k$ if $\pi_{2}\left(K_{\beta}^{i-1}(\omega, x)\right) \in C_{k}$.

Remark 5. The proof of the above theorem shows that $Q \circ \psi^{-1}$ is a $K_{\beta}$-invariant measure on $\Omega \times\left[0, b_{1} /(\beta-1)\right]$ with support $Z$, and of maximal entropy $\log (1+\lfloor\beta\rfloor)$. By Theorem 5 it follows that $Q \circ \psi^{-1}=v_{\beta}$. In Theorem 6 , the projection of this measure on the second coordinate was identified as an infinite convolution of Bernoulli measures.

Let $\pi_{1}: \Omega \times[0,\lfloor\beta\rfloor /(\beta-1)] \rightarrow \Omega$ be the canonical projection onto the first coordinate. Consider the measure $Q^{\prime}=\nu_{\beta} \circ \pi_{1}^{-1}$ on $\Omega$. Then $Q^{\prime}=Q \circ \alpha^{-1}$, where $\alpha=\pi_{1} \circ \psi: Y^{\prime} \rightarrow \Omega$.

Theorem 8. The measure $Q^{\prime}$ is the uniform Bernoulli measure on $\{0,1\}^{\mathbb{N}}$.

Proof. Define the stopping times $\left(T_{i}\right)_{i \geq 1}$ on $Y^{\prime}$ recursively as follows:

$$
\begin{aligned}
T_{1} & =\min \left\{m \geq 2: y_{m-1} \in\left\{s_{1}, \ldots, s_{b_{1}}\right\}\right\}, \\
T_{i} & =\min \left\{m>T_{i-1}: y_{m-1} \in\left\{s_{1}, \ldots, s_{b_{1}}\right\}\right\}, \quad i \geq 2 .
\end{aligned}
$$

An application of the Strong Markov Property shows that the stopped process $y_{T_{1}}, y_{T_{2}}, \ldots$ is also a Markov chain with state space $\{0, L\}$ and transition probabilities given by $q_{i j}=$ $1 / 2$ for $i, j \in\{0, L\}$. Therefore, if $j_{1}, \ldots, j_{l} \in\{0, L\}$, then

$$
Q\left(\left\{y_{T_{1}}=j_{1}, \ldots, y_{T_{l}}=j_{l}\right\}\right)=1 / 2^{l} .
$$

Define $\chi:\{0, L\} \rightarrow\{0,1\}$ by $\chi(0)=1, \chi(L)=0$. It follows that

$$
Q^{\prime}\left(\left\{\omega_{1}=\chi\left(j_{1}\right), \ldots, \omega_{l}=\chi\left(j_{l}\right)\right\}\right)=Q\left(\left\{y_{T_{1}}=j_{1}, \ldots, y_{T_{l}}=j_{l}\right\}\right)=1 / 2^{l} .
$$

Remarks 6. (1) If 1 has a finite greedy expansion $1=b_{1} / \beta+\cdots+b_{n} / \beta^{n}$ with some of the coefficients $b_{i}$ equal to zero, then one is able to find examples of such $\beta$ 's where the map $K_{\beta}$ has an underlying Markov partition similar to the one described above, i.e. determined by the random orbits of 1 and $b_{1} /(\beta-1)-1$. On the other hand, one is also able to find examples where $K_{\beta}$ has no such Markov partition. For example, for $n \geq 2$, let $\beta_{n} \in(1,2)$ be the unique solution to the equation

$$
\beta^{n}=\beta^{n-1}+1
$$


Then 1 has a greedy expansion $1=1 /(\beta)+1 /\left(\beta^{n}\right)$. For $n=2,3,4,5$, it is not hard to see that $K_{\beta}$ has a natural underlying Markov partition (one might need to divide the switch regions as well). However, for $n$ sufficiently large this is not the case. For in [EK] it was shown that for each $\beta$ sufficiently close to 1 , there exists a sequence $\left(\epsilon_{i}\right)$ of zeros and ones satisfying $\sum_{i=1}^{\infty} \epsilon_{i} / \beta^{i}=1$ and containing all possible finite variations of the digits 0 and 1. Now, it is easy to check that $\beta_{n} \downarrow 1$ as $n \rightarrow \infty$. Hence, if $\beta_{n}$ is sufficiently close to 1 , then by Theorem 2 there is an $\omega \in \Omega$ such that $\epsilon_{i}=d_{i}(\omega, 1)$ for each $i$. Since each block of zeros and ones appears in $\left(d_{i}(\omega, 1)\right)_{i \geq 1}$ this implies that

$$
\overline{\left\{\pi_{2}\left(K_{\beta_{n}}^{m}(\omega, 1)\right): m \geq 0\right\}}=\left[0, \frac{1}{\beta_{n}-1}\right] .
$$

Hence, there is no underlying Markov partition (determined by the random orbits of 1 and $\left.1 /\left(\beta_{n}-1\right)-1\right)$ for the map $K_{\beta}$.

Notice that $\beta_{5}$ is the smallest Pisot number. One might conjecture that for $\beta \in\left(1, \beta_{5}\right)$, one cannot construct a Markov partition similar to the one described in this section.

(2) We now consider the case $\beta^{2}=b_{1} \beta+1$. Notice that $\mathcal{C}=\mathcal{E}$, since 1 and $b_{1} /(\beta-1)$ -1 are already endpoints of intervals in $\mathcal{E}$. For ease of notation, we denote the alphabet of $Y$ by $\left\{e_{0}, s_{1}, e_{1}, \ldots, s_{b_{1}}, e_{b_{1}}\right\}$. For any $1 \leq i \leq b_{1}$,

$$
T_{\beta}\left(S_{i}\right)=\bar{E}_{0}=[0,1 / \beta], \quad S_{\beta}\left(S_{i}\right)=\bar{E}_{b_{1}}=\left[1, \frac{b_{1}}{\beta-1}\right] .
$$

As a result, Lemmas 6 and 7 do not hold for elements in $Y^{\prime}$ corresponding to endpoints of elements of $\mathcal{E}$. To be precise, for $1 \leq i \leq b_{1}$ we define the sequences $x^{(i)}, y^{(i)}, q^{(i)}$ and $r^{(i)}$ as follows.

- Let $x^{(i)}=\left(s_{i}, e_{b_{1}}, s_{1}, e_{b_{1}}, s_{1}, \ldots\right)$. Then $\psi\left(x^{(i)}\right)=\left(\omega^{(0)}, i / \beta\right)$, where $\omega^{(0)}=$ $(0,0,0, \ldots)$. We have $x_{2 m+1}^{(i)}=s_{1}$ for $m \geq 1$, while for $j \geq 2$,

$$
\pi_{2}\left(K_{\beta}^{(j)}\left(\omega^{(0)}, \frac{i}{\beta}\right)\right)=\frac{b_{1}}{\beta-1} .
$$

- Let $y^{(i)}=\left(e_{i}, s_{1}, e_{b_{1}}, s_{1}, e_{b_{1}}, \ldots\right)$. Then

$$
\psi\left(y^{(i)}\right)=\left(\omega^{(0)}, \frac{b_{1}}{\beta(\beta-1)}+\frac{i-1}{\beta}\right) .
$$

We have $y_{2 m}^{(i)}=s_{1}$ for $m \geq 1$, while for $j \geq 1$,

$$
\pi_{2}\left(K_{\beta}^{(j)}\left(\omega^{(0)}, \frac{b_{1}}{\beta(\beta-1)}+\frac{i-1}{\beta}\right)\right)=\frac{b_{1}}{\beta-1} .
$$

- Let $q^{(i)}=\left(e_{i-1}, s_{b_{1}}, e_{0}, s_{b_{1}}, e_{0}, \ldots\right)$. Then $\psi\left(q^{(i)}\right)=\left(\omega^{(1)}, i / \beta\right)$, where $\omega^{(1)}=$ $(1,1,1, \ldots)$. We have $q_{2 m}^{(i)}=s_{b_{1}}$ for $m \geq 1$, while for $j \geq 1$,

$$
\pi_{2}\left(K_{\beta}^{(j)}\left(\omega^{(1)}, \frac{i}{\beta}\right)\right)=0 .
$$


- Let $r^{(i)}=\left(s_{i}, e_{0}, s_{b_{1}}, e_{0}, s_{b_{1}}, \ldots\right)$. Then $\psi\left(r^{(i)}\right)=\left(\omega^{(1)}, \frac{b_{1}}{\beta(\beta-1)}+\frac{i-1}{\beta}\right)$. We have for $m \geq 1, r_{2 m+1}^{(i)}=s_{b_{1}}$, while for $j \geq 2$,

$$
\pi_{2}\left(K_{\beta}^{(j)}\left(\omega^{(1)}, \frac{b_{1}}{\beta(\beta-1)}+\frac{i-1}{\beta}\right)\right)=0 .
$$

Except for these points, the analysis used in this section remains valid. So, the only modification needed is the removal of a set of measure zero from the domain of $Y^{\prime}$, namely all points whose orbit under $\sigma_{Y}$ eventually equals $x^{(i)}, y^{(i)}, q^{(i)}$ or $r^{(i)}$ for some $i=1, \ldots, b_{1}$.

(3) Suppose in the switch regions we decide to flip a biased coin, with $0<\mathbb{P}$ (Heads) $=p<1$, in order to decide whether to use the greedy or the lazy map. The measure of maximal entropy discussed in this section does not reflect this fact. A natural invariant measure that preserves this property is obtained by considering the Markov measure $Q_{\lambda}$ on $Y$ with transition probabilities $p_{i, j}$, given by

$$
p_{i, j}= \begin{cases}\lambda\left(C_{i} \cap T_{\beta}^{-1} C_{j}\right) / \lambda\left(C_{i}\right) & \text { if } i \in \bigcup_{k=0}^{b_{1}} M_{k}, \\ p & \text { if } i \in\{0,1, \ldots, L\} \backslash \bigcup_{k=0}^{b_{1}} M_{k} \text { and } j=0, \\ 1-p & \text { if } i \in\{0,1, \ldots, L\} \backslash \bigcup_{k=0}^{b_{1}} M_{k} \text { and } j=L, \\ 0 & \text { if } i \in\{0,1, \ldots, L\} \backslash \bigcup_{k=0}^{b_{1}} M_{k} \text { and } j \neq 0, L,\end{cases}
$$

(as before, $\lambda$ denotes Lebesgue measure) and initial distribution the corresponding stationary distribution (see [DK2]). Another interesting feature is that the projection of $Q_{\lambda} \circ \psi^{-1}$ on the second coordinate for $p=1$ is the Parry measure $\mu_{\beta}$, and for $p=0$ it is the lazy measure $\rho_{\beta}$ (see Section 1 ).

\section{References}

[DK1] Dajani, K., Kraaikamp, C.: From greedy to lazy expansions, and their driving dynamics. Expo. Math. 20, 315-327 (2002) Zbl 1030.11035 MR 2003h:11089

[DK2] Dajani, K., Kraaikamp, C.: On random $\beta$-expansions. Ergodic Theory Dynam. Systems 23, 461-479 (2003)

[E1] Erdős, P.: On a family of symmetric Bernoulli convolutions. Amer. J. Math. 61, 974-976 (1939) Zbl 0022.35402 MR 0000311

[E2] Erdős, P.: On the smoothness properties of Bernoulli convolutions. Amer. J. Math. 62, 180-186 (1940) Zbl 66.0511.02 MR 0000858

[EJK] Erdős, P., Joó, I., Komornik, V.: Characterization of the unique expansions $1=$ $\sum_{i=1}^{\infty} q^{-n_{i}}$ and related problems. Bull. Soc. Math. France 118, 377-390 (1990) Zbl 0721.11005 MR 91j:11006

[EK] Erdős, P., Komornik, V.: Developments in non-integer bases. Acta Math. Hungar. 79, 57-83 (1998) Zbl 0906.11008 MR 99e:11132

[G] Gel'fond, A. O.: A common property of number systems. Izv. Akad. Nauk SSSR Ser. Mat. 23, 809-814 (1959) (in Russian) MR 0109817

[JS] Joó, I., Schnitzer, F. J.: Expansions with respect to non-integer bases. Grazer Math. Berichte 329 (1996) Zbl 0941.11003 MR 98e:11090 
[KL] Komornik, V., Loreti, P.: Subexpansions, superexpansions and uniqueness properties in non-integer bases. Period. Math. Hungar. 44, 197-218 (2002) Zbl 1017.11008 MR 2003i:11019

[MS] Mauldin, R. D., Simon, K.: The equivalence of some Bernoulli convolutions to Lebesgue measure. Proc. Amer. Math. Soc. 126, 2733-2736 (1998) Zbl 0912.28004 MR 98i:26009

[P] Parry, W.: On the $\beta$-expansions of real numbers. Acta Math. Acad. Sci. Hungar. 11, 401416 (1960) Zbl 0099.28103 MR 0142719

[PSS] Peres, Y., Schlag, W., Solomyak, B.: Sixty years of Bernoulli convolutions. In: Fractal Geometry and Stochastics II, Progr. Probab. 46, Birkhäuser, 39-65 (1998) Zbl 0961.42006 MR 2001m:42020

[R1] Rényi, A.: Representations for real numbers and their ergodic properties. Acta Math. Acad. Sci. Hungar. 8 (1957), 477-493. MR 0097374

[R2] Rényi, A.: On algorithms for the generation of real numbers. Magyar Tud. Akad. Mat. Fiz. Oszt. Kzl. 7, 265-293 (1957) MR 0097645

[S] Salem, R.: Algebraic Numbers and Fourier Analysis. Heath (1963) Zbl 0126.07802 MR 0157941

[So] Solomyak, B.: On the random series $\sum \pm \lambda^{i}$ (an Erdős problem). Ann. of Math. 142, 611625 (1995) Zbl 0837.28007 MR 97d:11125

[W] Walters, P.: An Introduction to Ergodic Theory. Springer, New York (1982) Zbl 0475.28009 MR 84e:28017 